\title{
PHYTOCHEMICAL INVESTIGATION AND MOLLUSCICIDAL ACTIVITY OF OREOPANAX RETICULATUM DONN
}

\author{
Abdel-Gawad M. M., El-Nahas H. A., El-Sayed M.M. and Abdel-Hameed, E. S. \\ Laboratory of Medicinal Chemistry, Theodor Bilharz Research Institute, Warrak El-Hadar, \\ Giza, Egypt
}

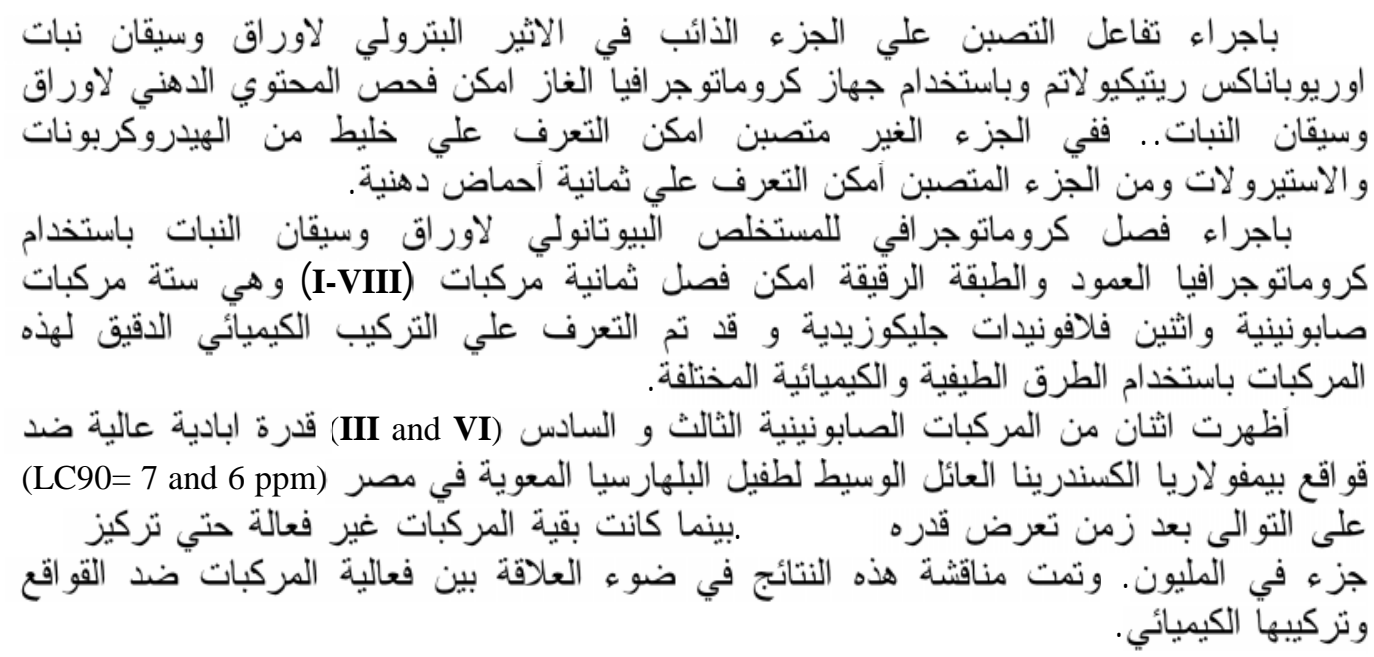

The unsaponifiable part of the petroleum ether fraction of the leaves and stems of Oreopanax reticulatum Donn (Family Araliaceae) was analysed by GLC to reveal the presence of a series of hydrocarbons, cholesterol, stigmasterol and $\beta$-sitosterol. Also, eight fatty acids were identified on the saponifiable part. The butanolic fraction was chromatographed over silica gel and sephadex-LH2O columns and preparative thin layer chromatography to afford two flavonoid glycosides (I and $\mathbf{I V})$ as well as four bidesmosidic triterpenoidal saponins (II, $\boldsymbol{V}$, VII and VIII). Alkaline hydrolysis of two bidesmosidic saponins (II and V) gave two monodesmosidic (III and VI). Their structures were elucidated using spectroscopic and chemical techniques.

Molluscicidal screening of the isolated compounds (I-VIII) against Biomphalaria alexandrina snails, the intermediate host of Schistosoma mansoni in Egypt revealed that the two monodesmosidic triterpenoidal saponins (III and VI) have strong activities $\left(L C_{90}=7\right.$ and 6 ppm) after 24 hours exposure whereas the two flavonoid glycosides and four bidesmosidic saponins were inactive up to $50 \mathrm{ppm}$.

\section{INTRODUCTION}

Schistosomiasis is still one of the most important parasitic diseases of man in Egypt and some tropical and subtropical parts of the world. ${ }^{1}$ The transmission of this disease requires vector snails as obligate intermediate hosts. Control of the vector snails is therefore, revelant to the control of this parasitic disease. $^{2,3}$ The use of plants with molluscicidal properties is a simple, inexpensive and appropriate technology for the snail control. ${ }^{4,5}$
Since the discovery of the active saponins in the berries of Phytolacca dodecandra, naturally occurring molluscicides are receiving considerable attention. ${ }^{5-7}$

In a previous study, it was observed that the water suspension of the leaves and stems of Oreopanax reticulatum (Family Araliaceae) has molluscicidal activity against Biomphalaria alexandrina snails, the intermediate host of Schistosoma mansoni in Egypt $\left(\mathrm{LC}_{90}=160\right.$ ppm) after 24 exposure. ${ }^{8}$ The available 
literatures revealed that nothing was reported on the chemistry of Oreopanax reticulatum. Meanwhile lupane and oleanane triterpenoidal saponins have been isolated from the other species; Oreopanax guatemalensis. ${ }^{9,10}$ This promoted us to isolate and identify the chemical constituents of $O$. reticulatum and evaluate the potency of the isolated compounds as molluscicidal agents against Biomphalaria alexandrina snails.

\section{EXPERIMENTAL}

\section{General}

Melting points were uncorrected.IR spectra were measured on a Perkin-Elmer model FT-IR recording spectrophotometer. Nuclear magnetic resonance ${ }^{1} \mathrm{H}-\mathrm{NMR}$ (270 $\mathrm{MHz})$ and ${ }^{13} \mathrm{C}-\mathrm{NMR}(100 \mathrm{MHz})$ spectra were done using TMS as internal standard, DMSO$\mathrm{d}_{6}$ as solvent and chemical shifts are given in (ppm) scale. UV spectrophotometer (Milton Roy 601). Mass spectra were measured on a Finnigan TSQ 700 GC/MC equipped with a Finnigan electrospray source (EI-MS and CIMS).TLC was carried out on silica gel 60 GF254 and the spots were visualized by spraying with $40 \% \mathrm{H}_{2} \mathrm{SO}_{4}$ followed by heating in oven at $120^{\circ}$. Column chromatography was carried out on silica gel 60 (sigma 28-200 mesh). Centrifugal chromatography device was used for performing preparative chromatographic separations. Sephadex LH-20 columns were performed using methanol as eluent. Paper Chromatography was performed on Whatmann paper No. 1 using descending technique and visualized with aniline phthalate.

GLC analysis of the unsaponifiable matter was carried out on a GCV Pye Unicam gas chromatograph equipped with a dual flame ionization detector and a dual channel reactor using a coiled glass column $(2.8 \mathrm{~m} \mathrm{x} 4 \mathrm{~mm})$ packed with diatomite $\mathrm{C}$ (100-120 mesh) and coated with $1 \%$ OV-17. Oven temperature was programmed at $10^{\circ} / \mathrm{min}$ from $70-270^{\circ}$ then run isothermally at $270^{\circ}$ for 25 minutes. Nitrogen flow was $30 \mathrm{ml} / \mathrm{min}$. Detector and injector temperatures were generally $300^{\circ}$ and $280^{\circ}$ respectively. Hydrogen and air flow rates were 33 and $330 \mathrm{ml} / \mathrm{min}$ respectively.

GLC analysis of the fatty acid methyl esters was performed adapting the previously mentioned conditions except the following. A coiled glass column (1.5 m x $4 \mathrm{~mm})$ packed with $10 \%$ polyethylene glycol Adipate (PEGA) on diatomite C (100-120 mesh). Nitrogen was used as a carrier gas at a flow rate of (30 $\mathrm{ml} / \mathrm{min}$ ). the oven temperature was programmed at $80^{\circ} / \mathrm{min}$ from $70^{\circ}$ to $190^{\circ}$, then kept isothermal at $190^{\circ}$ for $30^{\circ}$.

\section{Plant}

The leaves and stems of Oreopanax reticulatum Donn (Family Araliaceae) were collected from El-Zohrea Botanical Garden, Giza, Egypt in March 2001. The plant was identified by Mrs. Traes Labib, general manager and head of specialist for plant identification in El-Orman Botanical Garden, Giza, Egypt. A voucher specimen was deposited in our laboratory. The leaves and stems of this plant were dried and powdered by electric mill and kept for extraction process.

\section{Extraction and fractionation}

The air-dried powdered leaves and stems of $O$. reticulatum $(1.5 \mathrm{~kg})$ were extracted with methanol. The methanolic extract was evaporated using rotatory evaporator under reduced pressure to dryness (198 g) and defatted with petroleum ether $\left(60-80^{\circ}\right)$. The petroleum ether extract $(9 \mathrm{~g})$ was saponified with methanolic potassium hydroxide $(10 \%)$ for 6 hours. The unsaponifiable fraction (USM) and fatty acid methyl esters (FAME) were prepared $^{11,12}$ and analysed by GLC. Identification of the hydrocarbons and sterols was carried out by comparing their relative retention times with the same reference matters as well as the reported data in the literatures ${ }^{12,13}$ The quantitative estimation of each compound was carried out based on a peak area measurement relative to other peaks in the chromatogram. Results of GLC analysis were listed in Tables 1 and 2. 
Table 1: GLC analysis of the unsaponifiable fraction of lipid content of the leaves and stems of Oreopanax reticulatum.

\begin{tabular}{|c|c|c|l||}
\hline $\begin{array}{c}\text { Peak } \\
\text { No. }\end{array}$ & $\mathrm{RR}_{\mathrm{t}}$ & $\begin{array}{c}\text { Relative } \\
\%\end{array}$ & $\begin{array}{c}\text { Authentic hydrocarbons } \\
\text { and sterols }\end{array}$ \\
\hline 1 & 0.205 & 0.237 & n-Dodecane \\
2 & 0.216 & 0.289 & n-tridecane \\
3 & 0.243 & 0.124 & Unknown \\
4 & 0.252 & 0.201 & Unknown \\
5 & 0.265 & 0.470 & n-Tetradecane \\
6 & 0.281 & 1.616 & n-Pentadecane \\
7 & 0.296 & 0.149 & Unknown \\
8 & 0.312 & 0.256 & Unknown \\
9 & 0.336 & 8.723 & n-Hexadecane \\
10 & 0.365 & 0.243 & n-Heptadecane \\
11 & 0.384 & 0.106 & n-Octadecane \\
12 & 0.394 & 0.595 & n-Eicosane \\
13 & 0.417 & 0.167 & n-Heneicosane \\
14 & 0.434 & 0.306 & n-Docosane \\
15 & 0.461 & 0.200 & n-Tricosane \\
16 & 0.479 & 14.843 & n-Tetracosane \\
17 & 0.490 & 2.374 & n-Pentacosane \\
18 & 0.529 & 5.266 & n-Hexacosane \\
19 & 0.565 & 7.071 & n-Octacosane \\
20 & 0.638 & 0.139 & Squalene \\
21 & 0.665 & 0.633 & Unknown \\
22 & 0.789 & 4.284 & Cholesterol \\
23 & 0.911 & 17.663 & Stigmasterol \\
24 & 1 & 33.809 & $\beta$-sitosterol \\
\hline
\end{tabular}

$\mathrm{RR}_{\mathrm{t}}=$ Retention time relative to $\beta$-sitosterol $\mathrm{R}_{\mathrm{t}}=22.25$

Table 2: GLC analysis of the fatty acid methyl esters of the leaves and stems of Oreopanax reticulatum.

\begin{tabular}{||c|c|c|l||}
\hline $\begin{array}{c}\text { Peak } \\
\text { No. }\end{array}$ & $\mathrm{RR}_{\mathrm{t}}$ & $\begin{array}{c}\text { Relative } \\
\%\end{array}$ & $\begin{array}{c}\text { Authentic fatty acid } \\
\text { methyl esters }\end{array}$ \\
\hline 1 & 0.497 & 7.686 & Lauric acid \\
2 & 0.593 & 17.078 & Myristic acid \\
3 & 0.646 & 1.333 & Unknown \\
4 & 0.706 & 2.133 & Unknown \\
5 & 0.761 & 2.682 & Isopalmitic acid \\
6 & 0.829 & 13.200 & Palmitic acid \\
7 & 0.899 & 3.437 & Stearic acid \\
8 & 1 & 41.862 & Oleic acid \\
9 & 1.214 & 1.735 & Linoleic acid \\
10 & 1.382 & 8.853 & Arachidic acid \\
\hline
\end{tabular}

$\mathrm{RR}_{\mathrm{t}}=$ Retention time relative to Oleic acid $\mathrm{R}_{\mathrm{t}}=20.00$

Chromatographic separation of the butanolic fraction of $o$. reticulatum

The deffated methanolic extract was dissolved in water and successively extracted with $\mathrm{CHCl}_{3}$, EtOAc and n-butanol.The butanolic fraction $(45 \mathrm{~g})$ was subjected to open column chromatography $(120 \times 5 \mathrm{~cm})$ packed with silica gel 60 (70-230 mesh, Merck). Elution was started with petroleum ether followed by $\mathrm{CHCl}_{3}$, gradient mixtures of $\mathrm{CHCl}_{3:} \mathrm{MeOH}$ and ending with pure methanol. Five major fractions A-E were obtained by elution with different mixtures of $\mathrm{CHCl}_{3}$. $\mathrm{MeOH} ; 90: 10 ; 85: 15 ; 80: 20 ; 70: 30$ and 60:40 respectively. Each of these fractions was separately purified on Sephadex LH-20 column using $\mathrm{MeOH}$ as eluent agent. The Sephadex column products of each fraction were collected and separately subjected to preparative TLC. Fraction A gave compound I (using solvent system $\mathrm{CHCl}_{3}: \mathrm{MeOH}: \mathrm{H}_{2} \mathrm{O}$, 30:10:1). Fraction B yielded compounds II and IV (using solvent system $\mathrm{CHCl}_{3}: \mathrm{MeOH}: \mathrm{H}_{2} \mathrm{O}$, 60:30:5). Fraction $\mathbf{C}$ yielded compound $\mathbf{V}$ (solvent system n-BuOH:MeOH: $\mathrm{H}_{2} \mathrm{O} ; 5: 1: 1$ ). Fractions $\mathbf{D}$ and $\mathbf{E}$ gave compounds $\mathbf{V I I}$ and VIII (solvent system n-BuOH: $\mathrm{MeOH}: \mathrm{H}_{2} \mathrm{O}$; 5:3:2) respectively. Alkaline hydrolysis of compounds II and V gave compounds III and VI respectively.

\section{Isolated compounds}

Compound I, yellow powder, m.p 184-186 $\mathrm{R}_{\mathrm{f}} 0.47\left(\mathrm{CHCl}_{3}: \mathrm{MeOH}: \mathrm{H}_{2} \mathrm{O} ; 30: 10: 1\right)$. UV $\lambda_{\max }$ $(\mathrm{MeOH}) \mathrm{nm}$ 266, 320, 344; $\left(\mathrm{AlCl}_{3}\right)$ 272, 344, 397; $\left(\mathrm{AlCl}_{3} / \mathrm{HCl}\right)$ 272, 341, 395; (NaOAc) 266, 320,346 and $\left(\mathrm{NaOAc} / \mathrm{H}_{3} \mathrm{BO}_{3}\right) 264,324,345$. ${ }^{1} \mathrm{H}-\mathrm{NMR}$ (DMSO-d $\left.{ }_{6}\right) \delta$ at ppm: $8.20(5-\mathrm{OH})$, $7.79\left(2 \mathrm{H}, \mathrm{d}, \mathrm{J}=8.6 \mathrm{~Hz}, \mathrm{H}-2^{\prime}\right.$ and $\left.\mathrm{H}-6^{\prime}\right), 6.91$ $\left(2 \mathrm{H}, \mathrm{d}, \mathrm{J}=8.6 \mathrm{~Hz}, \mathrm{H}-3^{\prime}\right.$ and $\left.\mathrm{H}-5^{\prime}\right), 6.72(1 \mathrm{H}, \mathrm{d}$, $\mathrm{J}=2.5 \mathrm{~Hz}, \mathrm{H}-8), 6.42(1 \mathrm{H}, \mathrm{d}, \mathrm{J}=2.5 \mathrm{~Hz}, \mathrm{H}-6)$, $5.53\left(1 \mathrm{H}, \mathrm{d}, \mathrm{J}=2.5 \mathrm{~Hz}, \mathrm{H}-1^{\prime \prime}\right.$ of Rha), $5.31(1 \mathrm{H}$, $\left.\mathrm{d}, \mathrm{J}=2.5 \mathrm{~Hz}, \mathrm{H}-1^{\prime \prime \prime} \mathrm{Rha}\right), 5.16(1 \mathrm{H}, \mathrm{d}, \mathrm{J}=7.5$ $\mathrm{Hz}, \mathrm{H}-1^{\prime \prime}$ of $\left.\mathrm{Gal}\right), 1.14\left(3 \mathrm{H}, \mathrm{d}, \mathrm{J}=6.2 \mathrm{~Hz}, \mathrm{CH}_{3}\right.$ $\mathrm{Rha})$ and $0.82\left(3 \mathrm{H}, \mathrm{d}, \mathrm{J}=6.2 \mathrm{~Hz}, \mathrm{CH}_{3}-\mathrm{Rha}\right)$. CIMS; m/z $741.8[\mathrm{M}+\mathrm{H}]^{+}, 579.1\left[_{\mathrm{M}-\mathrm{Glc}]^{+} \text {and }}\right.$ 433.2 [M-Glc-Rha] ${ }^{+}, 287.2$ [M-Glc- 2 Rha] $^{+}$. ${ }^{13} \mathrm{C}$-NMR spectrum see Table (3).<smiles></smiles>

Compound I 
Table 3: ${ }^{13} \mathrm{C}$-NMR spectral data of compounds I and IV (in DMSO- $\mathrm{d}_{6}$; TMS as internal standard).

\begin{tabular}{|c|c|c|}
\hline $\begin{array}{c}\text { Aglycone } \\
\text { part }\end{array}$ & $\begin{array}{c}\text { Compound (I) } \\
\delta \mathrm{C}\end{array}$ & $\begin{array}{c}\text { Compound (IV) } \\
\delta \mathrm{C}\end{array}$ \\
\hline 2 & 157.9 & 158.30 \\
\hline 3 & 135.14 & 135.46 \\
\hline 4 & 177.8 & 177.90 \\
\hline 5 & 160.90 & 161.56 \\
\hline 6 & 99.22 & 99.19 \\
\hline 7 & 161.84 & 162.40 \\
\hline 8 & 95.00 & 95.27 \\
\hline 9 & 156.89 & 157.20 \\
\hline 10 & 106.59 & 106.70 \\
\hline $1^{\prime}$ & 120.37 & 120.50 \\
\hline $2^{\prime}$ & 131.28 & 131.30 \\
\hline $3^{\prime}$ & 116.36 & 116.27 \\
\hline $4^{\prime}$ & 160.30 & 160.0 \\
\hline $5^{\prime}$ & 116.36 & 116.27 \\
\hline $6^{\prime}$ & 131.28 & 131.30 \\
\hline \multicolumn{3}{|l|}{ Sugar moieties } \\
\hline & 3-O-Rha & 3-O-Gal \\
\hline $1^{\prime \prime}$ & 98.62 & 100.20 \\
\hline $2^{\prime \prime}$ & 70.55 & 73.82 \\
\hline $3^{\prime \prime}$ & 72.80 & 75.92 \\
\hline $4^{\prime \prime}$ & 81.82 & 70.20 \\
\hline $5^{\prime \prime}$ & 70.10 & 76.07 \\
\hline \multirow[t]{2}{*}{$6^{\prime \prime}$} & 18.19 & 68.22 \\
\hline & 7-O-Rha & 7-O-Rha \\
\hline $1^{\prime \prime \prime}$ & 100.24 & 100.32 \\
\hline $2^{\prime \prime \prime}$ & 69.97 & 70.40 \\
\hline $3^{\prime \prime \prime}$ & 71.20 & 71.36 \\
\hline $4^{\prime \prime \prime}$ & 71.68 & 71.76 \\
\hline $5^{\prime \prime \prime}$ & 69.70 & 69.76 \\
\hline \multirow[t]{2}{*}{$6^{\prime \prime \prime}$} & 18.50 & 18.58 \\
\hline & Glc $(1 \rightarrow 4$ ") Rha & Rha $\left(1 \rightarrow 6^{\prime \prime}\right)$ Gal \\
\hline $1^{\prime \prime \prime \prime I}$ & 102.59 & 101.69 \\
\hline $2^{\prime \prime \prime \prime}$ & 72.40 & 70.63 \\
\hline $3^{\prime \prime \prime \prime \prime}$ & 77.49 & 71.36 \\
\hline $4^{\prime \prime \prime \prime \prime}$ & 70.55 & 71.64 \\
\hline $5^{\prime \prime \prime \prime \prime}$ & 76.01 & 69.76 \\
\hline $6^{\prime \prime \prime \prime \prime}$ & 61.83 & 18.08 \\
\hline
\end{tabular}

Compound II, white powder, m.p 226-228 ${ }^{\circ}, \mathrm{R}_{\mathrm{f}}$ $0.63 \quad\left(\mathrm{CHCl}_{3}: \mathrm{MeOH}: \mathrm{H}_{2} \mathrm{O} ; \quad 60: 30: 5\right) \quad$ IR $v$ (KBr)max cm $\mathrm{cm}^{-1}$ 3420.2, 2939.9, 1735.7, 1639.5, 1386.6, 1066.2 and 914.0. ${ }^{1} \mathrm{H}-\mathrm{NMR}$ (DMSOd $\left.{ }_{6}\right) \delta$ 0.75-1.11 (each 3H, s, 7 x Me), $1.59(3 \mathrm{H}$, $\mathrm{d}, \mathrm{J}=6.4 \mathrm{~Hz}, \mathrm{Me}$ of Rha), $4.70(1 \mathrm{H}, \mathrm{d}, \mathrm{J}=7.7$ $\mathrm{Hz}, \mathrm{H}-1$ of Glc), $4.78(1 \mathrm{H}, \mathrm{d}, \mathrm{J}=6.2 \mathrm{~Hz}, \mathrm{H}-1$ of Ara), $5.16(1 \mathrm{H}, \mathrm{d}, \mathrm{J}=7.8 \mathrm{~Hz}, \mathrm{H}-1$ of Glc $), 5.22$ $(1 \mathrm{H}, \mathrm{d}, \mathrm{J}=1.6 \mathrm{~Hz}, \mathrm{H}-1$ of Rha) and $5.25(1 \mathrm{H}, \mathrm{br}$, s, H-12). CI-MS; m/z 1057 [M+H] ${ }^{+}, 908.9$ [M$\mathrm{Rha}^{+}, 894.6$ [M-Glc] $^{+}, 879.2$ [M-Glc-O] $^{+}, 851$ $\left[\mathrm{M}-\mathrm{Glc}-\mathrm{COO}^{-}\right]^{+}, \quad 749.7 \quad[\mathrm{M}-\mathrm{Glc}-\mathrm{Rha}]^{+}, \quad 705.5$

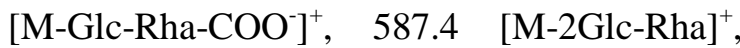
543.5 [M-2Glc-Rha-COO $]^{+}$, 455[M-2Glc-Rha$\mathrm{Ara}^{+}$, and 411.4 [M-2Glc-Rha-Ara-COO$]^{+}$. ${ }^{13} \mathrm{C}-\mathrm{NMR}$ spectrum see Tables 4 and 5 .

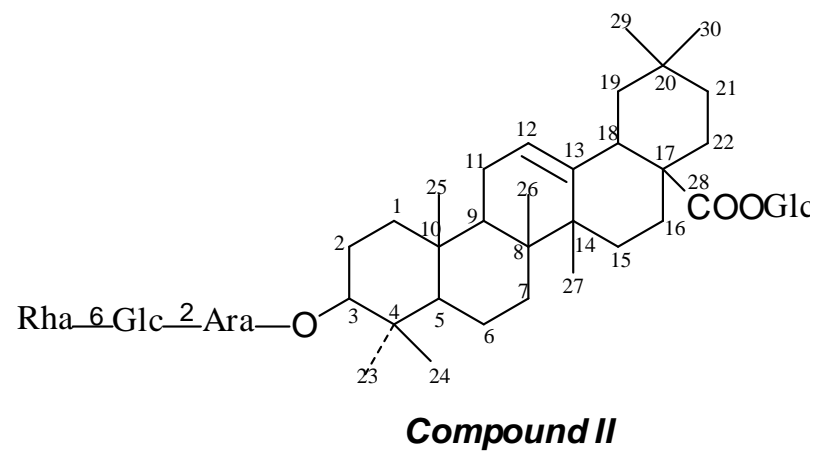

Compound III, white powder, m.p 244-247 $\mathrm{R}_{\mathrm{f}} 0.74\left(\mathrm{CHCl}_{3}: \mathrm{MeOH}: \mathrm{H}_{2} \mathrm{O}\right.$; 60: 30: 5). IR v $(\mathrm{KBr}) \max \quad \mathrm{cm}^{-1}$ 3434.5, 2931.9, 1691.4, 1462.2, 1387.9, 1078.1 and 999.1. ${ }^{1} \mathrm{H}-\mathrm{NMR}$ (DMSO-d ${ }_{6}$ ) $\delta$ 0.69-1.09 (each 3H, s, 7 x Me), $4.60(1 \mathrm{H}, \mathrm{d}, \mathrm{J}=7.9 \mathrm{~Hz}, \mathrm{H}-1$ of Glc $), 4.80(1 \mathrm{H}, \mathrm{d}$, $\mathrm{J}=6.4 \mathrm{~Hz}, \mathrm{H}-1$ of Ara $)$ and $5.18(1 \mathrm{H}, \mathrm{br}, \mathrm{s} \mathrm{H}-$ 12). CI-MS; m/z $751.7[\mathrm{M}+\mathrm{H}]^{+}, 704.7[\mathrm{M}+\mathrm{H}-$ $\mathrm{COOH}]^{+}, 587.5[\mathrm{M}+\mathrm{H}-\mathrm{Glc}]^{+}, 542.4[\mathrm{M}+\mathrm{H}-$ Glc-COOH] ${ }^{+}, 455.3$ [M-Glc-Ara] $^{+}$, and 411.2 $\left[\mathrm{M}-\mathrm{Glc}-\mathrm{Ara}-\mathrm{COO}^{-}\right]^{+} .{ }^{13} \mathrm{C}-\mathrm{NMR}$ spectrum see Tables 4 and 5 .<smiles>CC1(C)CCC2(C(=O)O)CC[C@]3(C)C(=CCC4C5(C)CCC(OC6CCCCC6)C(C)(C)C5CCC43C)C2C1</smiles>

Compound III 
Table 4: ${ }^{13} \mathrm{C}-\mathrm{NMR}$ spectral data of compounds II, III, V, VI, VII, VIII (in DMSO-d $\mathrm{d}_{6}$; TMS as internal standard).

\begin{tabular}{|c|c|c|c|c|c|c|}
\hline Aglycone & II & III & V & VI & VII & VIII \\
\hline 1 & 38.85 & 38.70 & 39.02 & 38.70 & 39.47 & 39.48 \\
2 & 26.36 & 26.1 & 26.18 & 26.30 & 28.17 & 26.17 \\
3 & 88.43 & 87.2 & 88.93 & 88.50 & 88.53 & 88.91 \\
4 & 39.73 & 38.80 & 39.45 & 39.20 & 39.74 & 39.76 \\
5 & 55.72 & 55.79 & 55.84 & 55.60 & 56.30 & 55.85 \\
6 & 18.43 & 18.39 & 18.50 & 18.70 & 18.43 & 18.45 \\
7 & 33.00 & 33.12 & 33.04 & 33.20 & 37.20 & 33.44 \\
8 & 39.73 & 39.80 & 39.72 & 39.50 & 40.29 & 39.48 \\
9 & 47.81 & 47.50 & 47.84 & 48.70 & 46.29 & 47.85 \\
10 & 37.03 & 36.90 & 36.96 & 39.20 & 38.40 & 36.97 \\
11 & 23.67 & 23.70 & 23.29 & 24.60 & 24.09 & 23.26 \\
12 & 122.04 & 121.90 & 122.41 & 123.2 & 131.40 & 122.44 \\
13 & 144.14 & 144.70 & 144.11 & 144.30 & 133.40 & 144.11 \\
14 & 42.4 & 42.3 & 42.01 & 42.60 & 56.32 & 42.02 \\
15 & 28.38 & 28.35 & 28.30 & 28.50 & 26.51 & 28.32 \\
16 & 24.10 & 24.5 & 24.08 & 26.70 & 26.17 & 24.10 \\
17 & 46.71 & 46.80 & 46.66 & 46.30 & 49.09 & 46.69 \\
18 & 42.04 & 42.16 & 41.50 & 42.50 & 54.60 & 41.15 \\
19 & 46.31 & 46.31 & 46.28 & 46.90 & 39.02 & 46.32 \\
20 & 30.97 & 30.97 & 30.30 & 31.2 & 36.02 & 30.97 \\
21 & 34.00 & 34.00 & 33.09 & 34.50 & 30.96 & 34.00 \\
22 & 33.01 & 33.01 & 33.44 & 33.70 & 36.02 & 33.44 \\
23 & 27.97 & 27.97 & 27.91 & 28.20 & 18.43 & 27.99 \\
24 & 17.14 & 17.14 & 17.42 & 16.80 & 28.27 & 17.41 \\
25 & 15.89 & 15.89 & 15.88 & 15.50 & 15.87 & 15.89 \\
26 & 17.14 & 17.14 & 17.79 & 17.40 & 18.43 & 17.10 \\
27 & 26.19 & 26.19 & 26.18 & 26.10 & 177.90 & 26.17 \\
28 & 175.97 & 179.85 & 176.49 & 179.80 & 175.96 & 176.06 \\
29 & 33.43 & 33.43 & 33.04 & 33.4 & 17.10 & 33.40 \\
30 & 23.67 & 23.67 & 23.66 & 23.6 & 20.80 & 23.65 \\
\hline
\end{tabular}


Table 5: ${ }^{13} \mathrm{C}-\mathrm{NMR}$ spectral data of sugar of compounds II, III, V, VI, VII, VIII (in DMSO-d $\mathrm{d}_{6}$; TMS as internal standard).

\begin{tabular}{|c|c|c|c|c|c|c|}
\hline & II & III & $\mathbf{V}$ & VI & VII & VIII \\
\hline & 3-O-Ara & 3-O-Ara & 3-O-GlcA & 3-O-GlcA & 3-O-Rha & 3-O-GlcA \\
\hline 1 & 106.38 & 106.20 & 104.30 & 104.20 & 101.30 & 103.34 \\
\hline 2 & 77.61 & 78.30 & 78.41 & 77.50 & 77.00 & 78.60 \\
\hline 3 & 76.21 & 76.34 & 82.15 & 82.20 & 83.59 & 82.91 \\
\hline 4 & 69.36 & 69.44 & 72.77 & 71.90 & 73.7 & 72.73 \\
\hline 5 & 65.36 & 65.20 & 74.23 & 74.70 & 69.34 & 74.50 \\
\hline 6 & -- & -- & 173.71 & 172.90 & 18.43 & 173.39 \\
\hline & Glc $(1 \rightarrow 2)$ Ara & Glc $(1 \rightarrow 2)$ Ara & Glc $(1 \rightarrow 3)$ GlcA & Glc $(1 \rightarrow 3)$ GlcA & Glc $(1 \rightarrow 3)$ Rha & Glc $(1 \rightarrow 2)$ GlcA \\
\hline 1 & 103.32 & 104.23 & 104.71 & 105.30 & 104.98 & 104.29 \\
\hline 2 & 75.97 & 76.30 & 74.23 & 74.40 & 72.97 & 74.50 \\
\hline 3 & 77.40 & 77.30 & 77.40 & 76.90 & 74.49 & 76.75 \\
\hline 4 & 71.75 & 72.40 & 70.33 & 70.50 & 70.68 & 71.50 \\
\hline 5 & 77.30 & 76.90 & 77.48 & 77.20 & 76.21 & 76.40 \\
\hline 6 & 69.36 & 61.89 & 61.47 & 61.80 & 61.73 & 61.31 \\
\hline & Rha $(1 \rightarrow 6)$ Glc & & Rha $(1 \rightarrow 2)$ GlcA & & 28-O-Glc & Xyl $(1 \rightarrow 3)$ GlcA \\
\hline 1 & 101.30 & & 101.40 & & 94.76 & 104.72 \\
\hline 2 & 72.70 & & 70.33 & & 77.52 & 76.8 \\
\hline 3 & 72.96 & & 72.77 & & 78.52 & 73.4 \\
\hline 4 & 73.41 & & 73.13 & & 71.38 & 69.2 \\
\hline 5 & 70.09 & & 70.33 & & 75.96 & 65.41 \\
\hline 6 & 17.45 & & 17.41 & & 60.73 & \\
\hline & 28-O-Glc & & 28-O-Glc & & & 28-O-Glc \\
\hline 1 & 94.76 & & 94.87 & & & 94.80 \\
\hline 2 & 73.41 & & 74.23 & & & 74.03 \\
\hline 3 & 77.40 & & 77.40 & & & 77.60 \\
\hline 4 & 70.09 & & 70.66 & & & 70.30 \\
\hline 5 & 77.40 & & 77.48 & & & 77.40 \\
\hline 6 & 61.39 & & 61.63 & & & 61.47 \\
\hline
\end{tabular}

Compound IV, yellow powder, m.p 202-205 ${ }^{\circ}$, $\mathrm{R}_{\mathrm{f}} 0.55\left(\mathrm{CHCl}_{3}: \mathrm{MeOH}: \mathrm{H}_{2} \mathrm{O} ; 60: 30: 5\right)$. UV $\lambda_{\max }$ (MeOH)nm 264, 325, 341; $\left(\mathrm{AlCl}_{3}\right)$ 272, 341, 395; $\left(\mathrm{AlCl}_{3} / \mathrm{HCl}\right) 272,341,392 ;(\mathrm{NaOAc}) 268$, 322,345 and $\left(\mathrm{NaOAc} / \mathrm{H}_{3} \mathrm{BO}_{3}\right) 268,324,343$. ${ }^{1} \mathrm{H}-\mathrm{NMR} \quad\left(\right.$ DMSO-d $\left._{6}\right) \quad \delta \quad 8.24 \quad(5-\mathrm{OH}), \quad 7.79$ $\left(1 \mathrm{H}, \mathrm{d}, \mathrm{J}=8.24 \mathrm{~Hz}, \mathrm{H}-2^{\prime}-\mathrm{H}^{\prime} 6^{\prime}\right), 6.94(2 \mathrm{H}, \mathrm{d}, \mathrm{J}=$ $\left.8.5 \mathrm{~Hz}, \mathrm{H}-3^{\prime}, \mathrm{H}-5^{\prime}\right), 6.78(1 \mathrm{H}, \mathrm{d}, \mathrm{J}=2.5 \mathrm{~Hz}, \mathrm{H}-$ 8), $6.42(1 \mathrm{H}, \mathrm{d} \mathrm{J}=2.5 \mathrm{~Hz}, \mathrm{H}-6), 5.57(1 \mathrm{H}, \mathrm{d}, \mathrm{J}=$ $2.5 \mathrm{~Hz}, \mathrm{H}-1^{\prime \prime \prime \prime}$ of Rha), $5.53(1 \mathrm{H}, \mathrm{d}, \mathrm{J}=2.5 \mathrm{~Hz}$, $\left.\mathrm{H}-1^{\prime \prime \prime} \mathrm{Rha}\right), 4.94\left(1 \mathrm{H}, \mathrm{d}, \mathrm{J}=7.6 \mathrm{~Hz}, \mathrm{H}-1^{\prime \prime}\right.$ of Gal), $1.15\left(3 \mathrm{H}, \mathrm{d}, \mathrm{J}=6.1 \mathrm{~Hz}, \mathrm{CH}_{3}-\mathrm{Rha}\right)$ and $0.85\left(3 \mathrm{H}, \mathrm{d}, \mathrm{J}=6.1 \mathrm{~Hz}, \mathrm{CH}_{3}-\mathrm{Rha}\right) \mathrm{CI}-\mathrm{MS} ; \mathrm{m} / \mathrm{z}$ $741.5[\mathrm{M}]^{+}, 595.3[\mathrm{M}-\mathrm{Rha}]^{+}, 433.5$ [M-Rha-
$\mathrm{Gal}^{+}$and 287 [M-2 Rha-Gal $]^{+} .{ }^{13} \mathrm{C}-\mathrm{NMR}$ spectrum see Table 3 .<smiles></smiles>

Compound IV 
Compound V, white powder, m.p 255-257 ${ }^{\circ}, \mathrm{R}_{\mathrm{f}}$ 0.58 (n-BuOH: $\mathrm{MeOH}: \mathrm{H}_{2} \mathrm{O}$; 5: 1: 1). IR $v$ (KBr)max cm $\mathrm{cm}^{-1} 3415.6$ 2943.2, 1732.7, 1617.4, 1417.8, 1077.9 and 950.9. ${ }^{1} \mathrm{H}-\mathrm{NMR}$ (DMSO$\left.\mathrm{d}_{6}\right) \delta$ 0.73-1.06 $\left(\mathrm{CH}_{3}, \mathrm{~s}, 7 \mathrm{x} \mathrm{Me}\right), 1.69(3 \mathrm{H}, \mathrm{d}$, $\mathrm{J}=6.2 \mathrm{~Hz}, \mathrm{CH}_{3}$ of Rha), $4.39(1 \mathrm{H}, \mathrm{d}, \mathrm{J}=7.6 \mathrm{~Hz}$, $\mathrm{H}-1$ of GlcA), $4.45(1 \mathrm{H}, \mathrm{d}, \mathrm{J}=7.6 \mathrm{~Hz}, \mathrm{H}-1$ of Glc), $5.14(1 \mathrm{H}, \mathrm{d}, \mathrm{J}=7.8 \mathrm{~Hz}, \mathrm{H}-1 \mathrm{Glc}), 5.21$ $(1 \mathrm{H}, \mathrm{d}, \mathrm{J}=1.6 \mathrm{~Hz}, \mathrm{H}-1$ of Rha) and $5.23(1 \mathrm{H}$, br,s,H-12). CI-MS; m/z $1101[\mathrm{M}+\mathrm{H}]^{+}$, 939.6 $[\mathrm{M}+\mathrm{H}-\mathrm{Glc}]^{+}, 893 \quad\left[\mathrm{M}+\mathrm{H}-\mathrm{Glc}-\mathrm{COO}^{+}\right]^{+}, \quad 793.7$ $[\mathrm{M}+\mathrm{H}-\mathrm{Glc}-\mathrm{Rha}]^{+}$, 748.1 [M-Glc-Rha-COO$]^{+}$, 633.6 [M-2Glc-Rha], 585.5 [M-2Glc-Rha$\mathrm{COO}^{-}{ }^{+}$and $455.4[\mathrm{M}-2 \mathrm{Glc}-\text { Rha-GlcA }]^{+} .{ }^{13} \mathrm{C}-$ NMR spectrum see Tables 4 and 5.

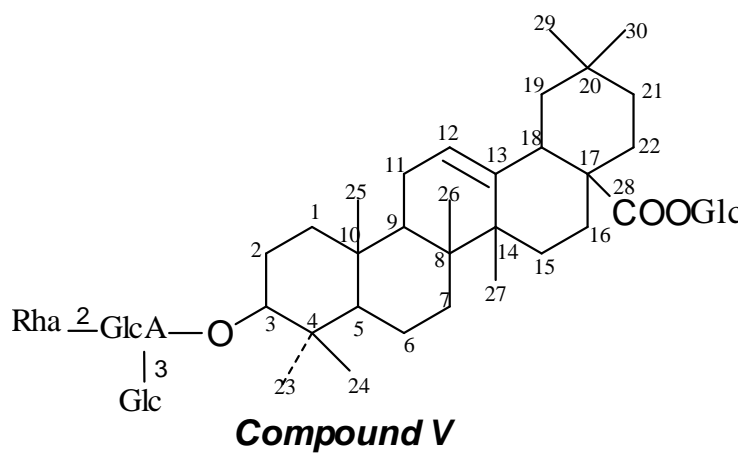

Compound VI, white powder m.p 248-250 ${ }^{\circ}$, $\mathrm{R}_{\mathrm{f}} 0.76$ (n-BuOH: MeOH: $\mathrm{H}_{2} \mathrm{O}$; 5: 1: 1). IR $v$ (KBr)max cm ${ }^{-1}$ 3419.5, 2930.7, 1695.5, 1461.6, 1167.4, 1078.2 and 978. ${ }^{1} \mathrm{H}-\mathrm{NMR}$ (DMSO-d ${ }_{6}$ ) $\delta$ 0.73-1.06 (each 3H, s, 7 x Me), $4.43(1 \mathrm{H}, \mathrm{d}$, $\mathrm{J}=7.7 \mathrm{~Hz}, \mathrm{H}-1$ of GlcA), $4.35(1 \mathrm{H}, \mathrm{d}, \mathrm{J}=7.8$ $\mathrm{Hz}, \mathrm{H}-1$ of Glc) and $5.22(1 \mathrm{H}, \mathrm{br}, \mathrm{s}, \mathrm{H}-12)$. CIMS; m/z $793.5[\mathrm{M}+\mathrm{H}]^{+}, 749.1[\mathrm{M}-\mathrm{COOH}]^{+}$, $631.2[\mathrm{M}+\mathrm{H}-\mathrm{Glc}]^{+}, 587.2[\mathrm{M}+\mathrm{H}-\mathrm{Glc}-\mathrm{COOH}]^{+}$ and 455.4 [M-Glc-GlcA] ${ }^{+}$. ${ }^{13} \mathrm{C}-\mathrm{NMR}$ spectrum see Tables 4 and 5 .

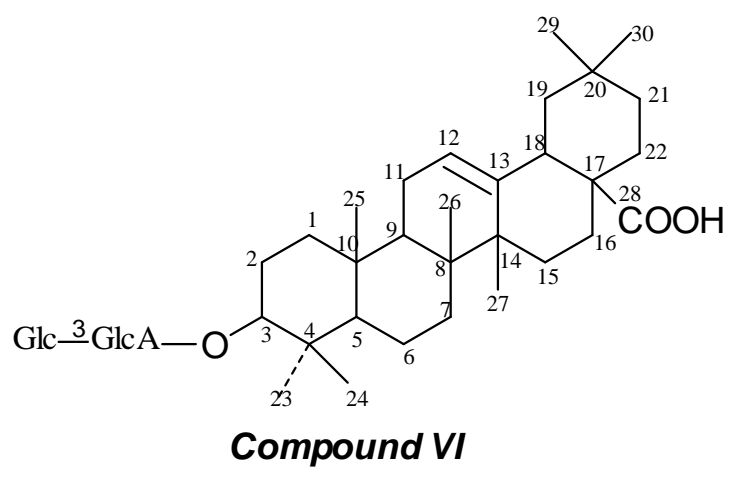

Compound VII, white powder m.p 223-225 $\mathrm{R}_{\mathrm{f}} 0.62$ (n-BuOH: $\mathrm{MeOH}: \mathrm{H}_{2} \mathrm{O} ; 5: 3: 2$ ). IR $v$ (KBr) $\operatorname{max~cm} \mathrm{cm}^{-1} 3387.9,2931.8,1734.3,1631$, 1446, 1129.3, 1072.7 and 929.4. ${ }^{1} \mathrm{H}-\mathrm{NMR}$ $\left(\right.$ DMSO-d $\left._{6}\right) \delta 0.69-1.06$ (each $\left.3 \mathrm{H}, \mathrm{s}, 6 \times \mathrm{Me}\right)$, $1.61\left(3 \mathrm{H}, \mathrm{d}, \mathrm{J}=6.5 \mathrm{~Hz}, \mathrm{CH}_{3}\right.$ of Rha), $4.65(1 \mathrm{H}$, $\mathrm{d}, \mathrm{J}=7.6 \mathrm{~Hz}, \mathrm{H}-1$ of $\mathrm{Glc}), 4.70(1 \mathrm{H}, \mathrm{d}, \mathrm{J}=7.9$ $\mathrm{Hz}, \mathrm{H}-1$ of Glc), $4.85(1 \mathrm{H}, \mathrm{d}, \mathrm{J}=1.65 \mathrm{~Hz}, \mathrm{H}-1$ of Rha) and $5.18(1 \mathrm{H}, \mathrm{br}, \mathrm{s}, \mathrm{H}-12)$. CI-MS; m/z $955.6[\mathrm{M}+\mathrm{H}]^{+}, \quad 911.7 \quad\left[\mathrm{M}+\mathrm{H}-\mathrm{COO}^{-}\right], \quad 793.9$ $[\mathrm{M}+\mathrm{H}-\mathrm{Glc}] \quad+, \quad 749.7 \quad\left[\mathrm{M}+\mathrm{H}-\mathrm{Glc}-\mathrm{COO}^{-}\right]^{+}$ $705.8\left[\mathrm{M}+\mathrm{H}-\mathrm{Glc}-2 \mathrm{COO}^{-}\right]^{+}, \quad 648.0 \quad[\mathrm{M}+\mathrm{H}-\mathrm{Glc}-$ $\mathrm{Rha}^{+}$, 604.1 [M-Glc-Rha-COO ${ }^{+}$and 485.4 $[\mathrm{M}-2 \text { Glc- Rha }]^{+}$. ${ }^{13} \mathrm{C}-\mathrm{NMR}$ spectrum see Tables 4 and 5 .

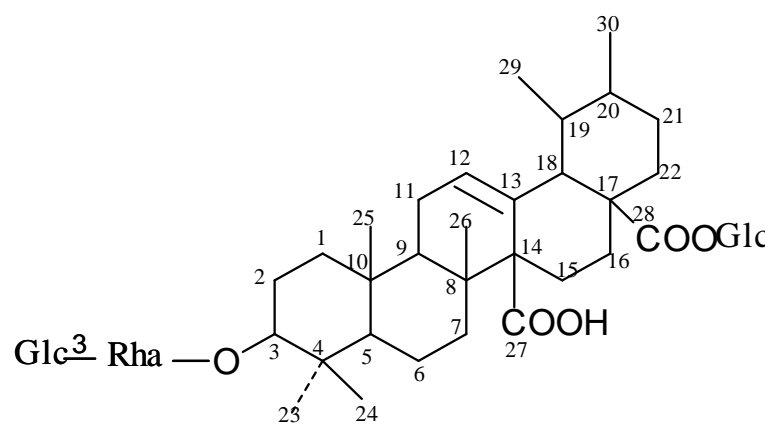

\section{Compound VII}

Compound VIII, white powder, m.p 232-234 ${ }^{\circ}$, $\mathrm{R}_{\mathrm{f}} 0.51$ (n-BuOH: $\mathrm{MeOH}: \mathrm{H}_{2} \mathrm{O} ; 5: 3: 2$ ). IR $v$ (KBr)max cm ${ }^{-1}$ 3422.2, 2927.1, 1732.8, 1602.2, 1386.0, 1078.1 and 940. ${ }^{1} \mathrm{H}-\mathrm{NMR}$ (DMSO-d $\mathrm{d}_{6}$ ) $\delta$ 0.72-1.09 (each 3H, s, 7 x Me), $4.69(1 \mathrm{H}, \mathrm{d}$, $\mathrm{J}=7.6 \mathrm{~Hz}, \mathrm{H}-1 \mathrm{GlcA}), 4.80(1 \mathrm{H}, \mathrm{d}, \mathrm{J}=7.8 \mathrm{~Hz}$, $\mathrm{H}-1$ of Glc), $5.15(1 \mathrm{H}, \mathrm{d}, \mathrm{J}=7.9 \mathrm{~Hz}, \mathrm{H}-1$ of Glc), $5.20(1 \mathrm{H}, \mathrm{d}, \mathrm{J}=6.9 \mathrm{~Hz}, \mathrm{H}-1$ of $\mathrm{Xyl})$ and $5.25(1 \mathrm{H}$, br, s, H-12). CI-MS; m/z 1087.2 $[\mathrm{M}+\mathrm{H}]^{+}, 925.8$ [M-Glc] $^{+}, 880.4$ [M-Glc-COO]$^{+}, 749.6[\mathrm{M} \text {-Glc- Xyl-COO }]^{+}, 719.8$ [M-2Glc$\left.\mathrm{COO}^{-}\right]^{+}, 618.7$ [M-Glc-Xyl-GlcA], 587.5 [M2Glc-Xyl-COO $]^{+}, 455.4$ [M-2Glc-Xyl-GlcA] and 411 [M-2Glc-Xyl-GlcA-COO']. ${ }^{13} \mathrm{C}-\mathrm{NMR}$ spectrum see Tables 4 and 5 .

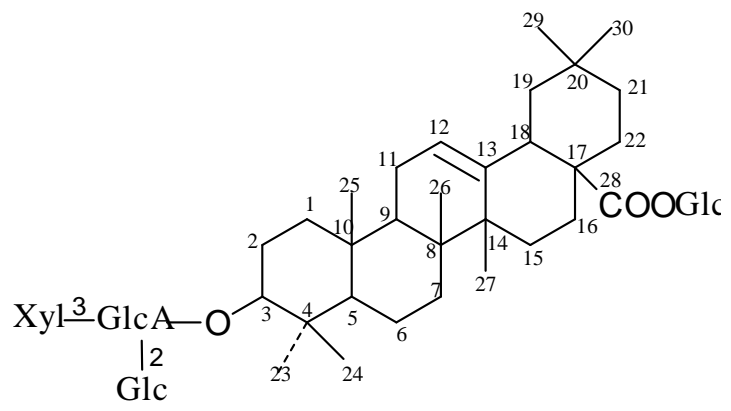

Compound VIII 


\section{Acid hydrolysis of saponins}

Each saponin $(20 \mathrm{mg})$ was refluxed with 4 $\mathrm{N} \mathrm{HCl}(30 \mathrm{ml})$ for 4 hours. The reaction mixture was diluted with water and extracted with chloroform. The chloroform extract was evaporated to dryness and the aglycone part was identified by TLC analysis with authentic samples in each case using solvent system $\mathrm{C}_{6} \mathrm{H}_{6}: \mathrm{MeOH},(8: 2)$. The aqueous layers were neutralized with $\mathrm{NaHCO}_{3}$, filtered, concentrated and compared with standard sugars on $\mathrm{PC}$ using solvent system (n-BuOH$\mathrm{AcOH}-\mathrm{H}_{2} \mathrm{O}$, 4:1:5). Spots were detected by spraying with a solution of aniline phthalate.

\section{Alkaline hydrolysis of saponins}

About $5 \mathrm{mg}$ of bidesmosidic saponins (II and $\mathbf{V}$ ) was refluxed with $5 \mathrm{ml} 1 \mathrm{M} \mathrm{NaOH}$ for 3 hours. The reaction mixture was neutralized and the prosapogenin was extracted with n$\mathrm{BuOH}$. The aqueous layer was subjected to paper chromatography to identify sugar part with authentic sugars.

\section{Molluscicidal Assay}

Biomphalaria alexandrina snails, the intermediate host of Schistosoma mansoni were collected from canals in Abu-Rawash, Giza Governorate. The snails were maintained in dechlorinated tap water in the laboratory conditions (Temp 25 $\pm 2^{\circ}, \mathrm{pH}$ 7-7.7). Series of dilutions were prepared from each isolated compound to calculate the $\mathrm{LC}_{90}$ values. Ten snails were added in each concentration and the exposure time was 24 hours followed by 24 hours as recovery period. Three replicates were carried out for each case. Procedures and statistical analysis of data were carried out according to WHO and Litchfield and Wilcoxon procedures ${ }^{14,15}$

\section{RESULTS AND DISCUSSION}

The methanolic extract of the leaves and stems of $O$. reticulaum was defatted with pet.ether and the residue was dissolved in water and successively extracted with $\mathrm{CHCl}_{3}$, EtOAc and $\mathrm{n}-\mathrm{BuOH}$.

The petroleum ether extract was saponified and each of USM and FAME was analysed by GLC. From Table (1), it was concluded that the major hydrocarbons of the unsaponifiable fraction of $O$. reticulatum is nTetracosane (14.843\%) followed by nHexadecane (8.723\%), n-Octacosane (7.071\%), n-Hexacosane $(5.266 \%)$ and n-Pentacosane $(2.374 \%)$. The sterol part was represented by three components, cholesterol, stigmasterol and $\beta$-sitosterol where $\beta$-sitosterol was the highest percent $(33.809 \%)$.

GLC analysis of the fatty acid methyl esters (Table 2) of $O$. reticulatum showed the presence of ten fatty acids, where eight of them were identified. Oleic acid recorded high percent $(41.862 \%)$ whereas linoleic acid was the lowest one $(1.735 \%)$.

The butanolic layer was chromatographed using silica gel and sephadex columns and preparative LC using different solvent systems. The structure of the following isolated compounds were elucidated through spectroscopic analysis as IR, ${ }^{1} \mathrm{H}-\mathrm{NMR},{ }^{13} \mathrm{H}-$ NMR and CI-MS and identification of the product of acidic and alkaline hydrolysis of most of these compounds.

Compound I, gave positive tests for flavonoid glycosides. Its UV absorption bands with methanol and different reagents were similar to those for kampferol with substitution at $3-\mathrm{OH}$ and $7-\mathrm{OH}^{16-18}$ This was confirmed by the presence of the two signals in ${ }^{1} \mathrm{H}-\mathrm{NMR}$ at upfield $\delta 7.79\left(2 \mathrm{H}, \mathrm{d}, \mathrm{H}-2^{/ /} \mathrm{H}-6^{\prime}\right)$ and $6.91(2-\mathrm{H}$, $\left.\mathrm{d}, \mathrm{H}-3^{\prime}, \mathrm{H}-5^{\prime}\right)$ than original kampferol indicated the presence of substitution at 3-OH. Also, the presence of characteristic signals of H-6 and H8 at downfield $\delta 6.72(1 \mathrm{H}, \mathrm{d}, \mathrm{H}-8)$ and 6.42 $(1 \mathrm{H}, \mathrm{d}, \mathrm{H}-6)$ than the usual pattern in kampferol ( $\delta 6.5$ for $\mathrm{H}-8$ and 6.1 for $\mathrm{H}-6$ ) indicated the presence of sugar substitution at C-7. ${ }^{19,20}$ Appearance of two signals as doublets at $\delta 5.53$ $(\mathrm{H}-1)$ and $5.31(\mathrm{H}-1)$ indicated the presence of two rhamnosyl units substituted at 3-OH and 7$\mathrm{OH}$ respectively. This was supported by appearance of two methyl groups of two rhamnose units at $\delta 0.82$ and $1.14 .{ }^{19,20}$ The ${ }^{13} \mathrm{C}$ NMR spectrum of this compound (Table 3 ) showed the characteristic signals of ketonic carbon at $\delta$ 177.8, acidic carbon $\mathrm{C}-7$ at $\delta$ $161.84, \mathrm{C}-5$ at $160.90, \mathrm{C}-4^{\prime}$ at $\delta 160.30, \mathrm{C}-2$ at $\delta 157.9$ and C-9 at 156.89. Also, signal of C-3 appeared at $\delta 135.14$ followed by nonoxygenated carbons $\mathrm{C}-2^{\prime}$ and $\mathrm{C}-6^{\prime}$ at $\delta 131.28$ as well as $\mathrm{C}-3^{\prime}$ and $\mathrm{C}-5^{\prime}$ at $\delta 116.36$. The most non-affected quaternary carbons $\mathrm{C}-1^{\prime}$ appeared 
at $\delta 120.37$ and $\mathrm{C}-10$ at $\delta 106.59 .^{20-23}$ The signals of two carbons C-6 and C-8 appeared at $\delta 99.22$ and 95.0 respectively. The anomeric carbons of the sugar units appeared at $\delta 98.62$, 102.59 and 100.24 . This was supported by the presence of three anomeric protons of sugar units in ${ }^{1} \mathrm{H}-\mathrm{NMR}$ at $\delta 5.53,5.31$ and 5.16. Also, the two-methyl signals of two rhamnose units appeared at $\delta 18.19$ and 18.50. The downfield shift of $\mathrm{C}-4^{\prime \prime}$ of rhamnose unit at $\delta 81.82^{\prime \prime}$ indicated that the terminal glucose unit is connected at this carbon, ${ }^{21,22}$ CI-MS spectrum of compound $\mathbf{I}$, gave $\mathrm{m} / \mathrm{z} 741.8\left[\mathrm{M}^{+}\right], 579.1$

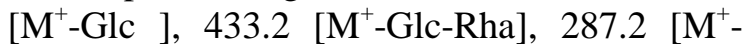
Glc-2Rha]. ${ }^{22,23}$ From the above data, the structure of compound I was elucidated as kampferol-3-O-[glucosyl $\left(1 \rightarrow 4^{\prime /}\right)$ rhamnoside]7-O-rhamnoside.

Compound II, its IR spectrum demonstrated the presence of hydroxyl group $\left(3420.2 \mathrm{~cm}^{-1}\right)$, trisubstituted double bond $\left(1639.5 \mathrm{~cm}^{-1}\right)$, ester group at $\left(1735.7 \mathrm{~cm}^{-1}\right)$ and glycosidic linkage at $1066.0 \mathrm{~cm}^{-1} \cdot{ }^{24,25}{ }^{1} \mathrm{H}-\mathrm{NMR}$ spectrum of compound II showed signals for seven methyl groups between a range $\delta$ 0.75-1.11, one trisubstituted olefinic proton at $\delta 5.25$ and four anomeric protons at $\delta 4.70,4.78,5.16$ and $5.22 .^{26,27}$ This was supported by presence of four anomeric carbons in ${ }^{13} \mathrm{C}$-NMR spectrum (Table 5) at $\delta 106.38,103.32,101.30$ and 94.76. ${ }^{26,28}$ The mass spectrum of compound II revealed a diagnostically two important mass peaks at $\mathrm{m} / \mathrm{z} 908.9$ [M-Rha] $^{+}$and $\mathrm{m} / \mathrm{z} 894.6$ $[\mathrm{M}-\mathrm{Glc}]^{+}$indicating its molecular weight was 1056. Other fragments ions at $\mathrm{m} / \mathrm{z} 851.0$ [MGlc-COO$\left.^{-}\right]^{+}, 749.7$ [M-Glc-Rha ${ }^{+}, 705.5$ [MGlc- Rha-COO' $]^{+}$, 587.4 [M-2Glc-Rha ${ }^{+}, 543.5$

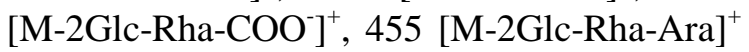
and 411.4 [M-2Glc-Rha-Ara-COO $]^{+}$indicated the respective elimination of two terminal sugar units, one rhamnose and one glucose as well as two other saccharide units, one glucose and one arabinose and indicated that the arabinose unit is linked to the aglycone part. ${ }^{26-}$ ${ }^{29}$ The signals of the aglycone part in ${ }^{13} \mathrm{C}-\mathrm{NMR}$ (Table 4) spectrum were identical with oleanolic acid as reported in the literature except for the signals of C-3 and C-28 which at downfield $\delta 88.43$ and $175.97 .^{26,27}$ This was supported by the upfield shift of one anomeric carbon at $\delta 94.76$ (Table 5) indicated esterification of the carboxyl group with sugar. ${ }^{26-28}$ Therefore, compound II was consequently a bidesmoside triterpenoid saponin. Alkaline hydrolysis of compound II gave compound III (as prosapogenin) as well as D-glucose and L-rhamnose as sugar moieties which were identified by comparison with authentic sugars on $\mathrm{PC}$ using solvent system $\mathrm{n}$ $\mathrm{BuOH}: \mathrm{AcOH}: \mathrm{H}_{2} \mathrm{O}$; (4:1:5). The signals of C2 of arabinose and C- 6 of the glucose units (Table 5) appeared at downfield $\delta 77.61$ and 69.36 indicating that the two carbons are the position of linkages between the sugar units. $^{26,29}$ Therefore, the structure of II was elucidated as 3-O- $\alpha$-L-rhamnopyranosyl$(1 \rightarrow 6)-\beta$-D-glucopyranosyl- $(1 \rightarrow 2)-\alpha-\mathrm{L}-$

arabinopyranosyl-oleanolic acid-28-O- $\beta$-Dglucopyranoside.

Compound III was obtained from alkaline hydrolysis of compound II. Its IR spectrum showed an absorption bands of hydroxyl groups at $3434.5 \mathrm{~cm}^{-1}$, carboxylic group at $1691.4 \mathrm{~cm}^{-1}$ and the characteristic band of the glycosidic linkage at $1078.1 \mathrm{~cm}^{-1}(24,25)$. The predominant fragments in CI-MS at $\mathrm{m} / \mathrm{z} 751.7$ $[\mathrm{M}+\mathrm{H}]^{+}, 704.7[\mathrm{M}-\mathrm{COOH}]^{+}, 587.5[\mathrm{M}-\mathrm{Glc}]^{+}$,

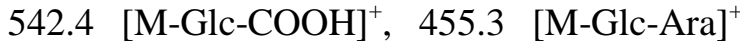
and $411.2 \quad[\mathrm{M}-\mathrm{Glc}-\mathrm{Ara}-\mathrm{COOH}]^{+}$. This fragmentation pattern reflected the subsequent loss of glucosyl and arabinosyl units. ${ }^{30,31}$ Also, the peak ascribable to the aglycone was observed $\mathrm{m} / \mathrm{z}$ 455.3. Comparison of ${ }^{1} \mathrm{H}$ and ${ }^{13} \mathrm{C}$ NMR data of compound III (Table 4 and 5) with those of compound II indicated that the aglycone part is the same (oleanolic acid) and the saccharide chain at C-3 lost of the rhamnosyl unit. Also, the saccharide chain at C-28 was absent where the signal C-28 was shifted at downfield $\delta 179.85$ indicating that compound III is monodesmosidic triterpenoidal glycoside. ${ }^{29,31}$ The ${ }^{1} \mathrm{H}$ and ${ }^{13} \mathrm{C}$ NMR spectra showed the characteristic signals of the two anomeric protons at $\delta 4.60$ and 4.80 and two anomeric carbons (Table 5) at $\delta$ 106.20 and 104.23 indicating that this compound has two sugar units. ${ }^{26,29}$ The signal of C-2 of arabinose unit shifted at downfield $\delta$ 78.30 (Table 5) reflecting that this carbon is the position of the interglycosidic linkage between the two sugar units. ${ }^{26,31}$ On the basis of these evidence, compound III was identified as 3-O$\beta$-D-glucopyranosyl-( $(1 \rightarrow 2)-\alpha$-L-arabinopyranosyl oleanolic acid. 
Compound IV, its UV spectrum with methanol and different reagents exhibited all the characteristic absorption bands of kampferol with 3-OH and 7-OH substitutions. ${ }^{17,18}$ This was confirmed by the appearing of two signals at upfield $\delta 7.79(2 \mathrm{H}$, $\left.\mathrm{d}, \mathrm{H}-2^{\prime}, 6^{\prime}\right)$ and $6.94\left(2 \mathrm{H}, \mathrm{d}, \mathrm{H}-3^{\prime}, 5^{\prime}\right)$ in the ${ }^{1} \mathrm{H}-$ NMR spectrum than the original kampferol indicating the presence of substitution at C-3. Also, two signals at downfield $\delta 6.78(1 \mathrm{H}, \mathrm{d}, \mathrm{H}-$ 8) and $6.42(1 \mathrm{H}, \mathrm{d}, \mathrm{H}-6)$ than kampferol suggesting the substitution at $\mathrm{C}-7 .{ }^{32,33}$ In ${ }^{13} \mathrm{C}$ NMR spectrum (Table 3 ), the two signals of $\mathrm{C}$ 3 and C-7 appearing at $\delta 135.46$ and 162.40 than kampferol indicating the substitution of these carbon atoms. ${ }^{19,33}$ CI-MS spectrum of compound IV exhibited a molecular ion at $\mathrm{m} / \mathrm{z}$ 741.5 and other fragment peaks at $\mathrm{m} / \mathrm{z} 595.3$ [ $\mathrm{M}^{+}$-Rha], 433.5 [ $\mathrm{M}^{+}$-Rha-Gal] and $287\left[\mathrm{M}^{+}\right.$2Rha-Gal] suggesting that compound IV has one galactosyl and two rhamnosyl units. ${ }^{22,23}$ This was supported by presence of three signals of anomeric protons at $\delta 5.57,5.53$ and 4.94 as well as two methyl signals of the two rhamnosyl units at $\delta 1.15$ and 0.85 . Also, in ${ }^{13} \mathrm{C}-\mathrm{NMR}$ spectrum (Table 3 ) the signals at the three anomeric carbon atoms were seen at $\delta$ 100.20, 101.69 and 100.32. The signal of C- 6 " of the galactosyl unit was shifted at downfield $\delta 68.22$ indicating the position of attachment of the terminal rhamnose of this carbon. ${ }^{22,23}$ From the above data compound IV was consistent with the structure kampferol 3-O-[rhamnosyl $\left(1 \rightarrow 6^{\prime \prime}\right)$ galactoside]-7-O-rhamnoside.

Compound $\mathbf{V}$, exhibited a molecular ion peak in CI-MS at m/z 1101.2 which in conjunction with the analysis of ${ }^{13} \mathrm{C}$-NMR spectrum shown (Table 4,5 ) to correspond to the molecular weight 1101. IR spectrum showed the characteristic signals for hydroxyl groups $3415.6 \mathrm{~cm}^{-1}$ and ester groups $1732.7 \mathrm{~cm}^{-1} .^{24,25}$ The ${ }^{1} \mathrm{H}-\mathrm{NMR}$ spectrum revealed the presence of seven tertiary methyl groups between $\delta 0.73$ and 1.06 , a double bond at $\delta 5.23$ with typical ${ }^{13} \mathrm{C}$-NMR resonances (Table 4 ) at $\delta 122.41$ and 144.11 indicating an olean-12-ene triterpene. $^{25,29}$ In the CI-MS of compound $\mathbf{V}$, the sequence of the sugar was established from diagnostic ions at $\mathrm{m} / \mathrm{z} 939.6[\mathrm{M}+\mathrm{H}-\mathrm{Glc}]^{+}, 893$ $\left[\mathrm{M}+\mathrm{H}_{-G l c-C O O}\right]^{+}, \quad 793.7 \quad[\mathrm{M}+\mathrm{H}-\mathrm{Glc}-\mathrm{Rha}]^{+}$, $748.1 \quad\left[\mathrm{M}+\mathrm{H}-\mathrm{Glc}-\mathrm{Rha}-\mathrm{COO}^{-}\right]^{+}, 633.6 \quad[\mathrm{M}+\mathrm{H}-$

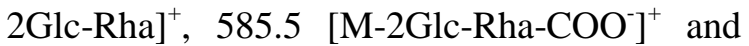

$455.4 \quad\left[\mathrm{M}^{-}-2 \mathrm{Glc}-\mathrm{Rha}-\mathrm{GlcA}\right]^{+24,29}$ On acid hydrolysis, compound $\mathbf{V}$ liberated oleanolic acid as the genin which was identified with an authentic sample by Co-TLC, IR and NMR. Also, the sugar moieties were D-glucuronic acid, D-glucose and L-rhamnose while alkaline hydrolysis of compound $\mathbf{V}$ gave glucose and rhamnose. ${ }^{25,30}$ In ${ }^{13} \mathrm{C}$-NMR spectrum (Table 4), the glycosidation shifts were observed at C-3 and $\mathrm{C}-28$ of the aglycone at $\delta 88.93$ and 176.49 respectively $^{24,30}$ indicating that the two sugar chains were attached to these positions. The ${ }^{1} \mathrm{H}-\mathrm{NMR}$ and ${ }^{13} \mathrm{C}-\mathrm{NMR}$ spectra of compound $\mathbf{V}$ showed signals for four anomeric protons at $\delta$ $4.39,4.45,5.14$ and 5.21 and four anomeric carbons (Table 5) at $\delta$ 104.30, 104.71, 101.40 and 94.87 respectively. ${ }^{29,30}$ The interglycosidic linkages between the sugar moiety was established by ${ }^{13} \mathrm{C}-\mathrm{NMR}$ spectrum (Table 5) where $\mathrm{C}-2$ and $\mathrm{C}-3$ of glucuronic acid were shifted at downfield $\delta 78.41$ and 82.15 indicating the two carbons were positions of attachments of the terminal sugar units, glucose and rhamnose. ${ }^{28-30}$ Thus the structure of compound $\mathbf{V}$ was assignated as $3-\mathrm{O}-\alpha-\mathrm{L}-$ rhamnopyranosyl-( $1 \rightarrow 2)$-[ $\beta$-D-glucopyranosyl$(1 \rightarrow 3)]-\beta$-D-glucuronopyranosyl oleanolic acid-28-O- $\beta$-D-glucopyranoside.

Compound VI was obtained from alkaline hydrolysis of compound V. Its ${ }^{13} \mathrm{C}-\mathrm{NMR}$ spectrum (Table 4 and 5) showed 42 signals, of which 30 were assigned to a triterpenoid moiety and 12 to a saccharide moiety. ${ }^{27,29}$ IR spectrum of this saponin reflected the characteristic band of carboxylic group at $1695.5 \mathrm{~cm}^{-1}$ as well as bands for hydroxyl group at $3419.5 \mathrm{~cm}^{-1}$ and glycosidic linkage at $1078.2 \mathrm{~cm}^{-1} .^{24,29}$ This was supported by the resonances of C-3 and C- 28 of the aglycone part of this saponin in ${ }^{13} \mathrm{C}-\mathrm{NMR}$ spectrum (Table 4) at $\delta 88.50$ and at $\delta 179.80$ which reflected the fact that the sugar chain was linked to $\mathrm{C}-3$ only ${ }^{28,29}$ The ${ }^{1} \mathrm{H}$ and ${ }^{13} \mathrm{C}-\mathrm{NMR}$ spectra of this compound exhibited two anomeric protons located at $\delta 4.43$ and 4.35 and two anomeric carbons (Table 5) at $\delta$ 104.20 and $105.30 .^{24,27}$ These data confirmed the sugar chain consists of two sugar units and this was supported by fragmentation pattern of this compound in CI-MS at $\mathrm{m} / \mathrm{z} 793.5[\mathrm{M}+\mathrm{H}$ ]$^{+}, 749.1$ [M-COO$\left.^{-}\right]^{+}, 631.2[\mathrm{M}+\mathrm{H}-\mathrm{Glc}]^{+}, 587.2$ $\left[\mathrm{M}+\mathrm{H}-\mathrm{Glc}-\mathrm{COO}^{-}\right]^{+}$and $455.2 \quad[\mathrm{M}+\mathrm{H}-\mathrm{Glc}-$ 
GlcA]. From the ${ }^{13} \mathrm{C}$-NMR spectrum (Table 5) it was noticed that the C-3 of glucuronic acid was shifted at downfield $\delta 82.20$ indicating that this carbon is the position of interglycosidic linkage between the sugar units. ${ }^{24-27}$ From the above data the structure of compound VI was deduced as 3-O- $\beta$-D-glucopyranosyl- $(1 \rightarrow 3)-\beta$ D-glucuronopyranosyl oleanolic acid.

Compound VII, showed a molecular peak in its CI-MS spectrum at $\mathrm{m} / \mathrm{z} 955.6[\mathrm{M}+\mathrm{H}]^{+}$. The predominant fragments at $\mathrm{m} / \mathrm{z} 911.7[\mathrm{M}+\mathrm{H}-$ $\left.\mathrm{COO}^{-}\right]^{+}, 793.9[\mathrm{M}+\mathrm{H}-\mathrm{Glc}]^{+}$and $749.7[\mathrm{M}+\mathrm{H}-$ Glc-COO$]^{+}$suggesting the subsequent loss of a glucose unit with and without the ester glycosidic linkage. Other fragments at $\mathrm{m} / \mathrm{z}$ 705.8 [M+H-Glc- $\left.2 \mathrm{COO}^{-}\right]^{+}, 648.0[\mathrm{M}+\mathrm{H}-\mathrm{Glc}-$ $\mathrm{Rha}^{+}$and $485.4[\mathrm{M}+\mathrm{H}-2 \mathrm{Glc}-\mathrm{Rha}]^{+}$reflected the lossing of two glucose units and one rhamnose unit. ${ }^{34}$ This was confirmed by presence of three anomeric protons in ${ }^{1} \mathrm{H}-\mathrm{NMR}$ spectrum at $\delta 4.65,4.70$ and 4.85 as well as appearing of three anomeric carbon atoms at $\delta$ 101.30, 104.98 and 94.76 in ${ }^{13} \mathrm{C}-\mathrm{NMR}$ spectrum as shown in Table 5..$^{35,36}$ Acid hydrolysis of compound VII yielded glucose and rhamnose as sugar moiety. A comparison of ${ }^{13} \mathrm{C}-\mathrm{NMR}$ spectrum of compound VII (Table 4) with the literature ${ }^{34,36}$ revealed that this compound has quinovic acid as the aglycone part with two glycosidation sides at C-3 and C$28 .^{35}$ The first glycosidation side was confirmed by shifting of C-3 at downfield $\delta$ 88.53 in the ${ }^{13} \mathrm{C}$-NMR spectrum (Table 4) compared with the same carbon of quinovic acid which appeared at $\delta 79.96 .^{35,36}$ Also, the other side was settled by shifting of C-28 at downfield $\delta 175.96$ as well as by the upfield shift of the anomeric carbon of one glucose unit at $\delta 94.76$ (Table 5). Its IR spectrum showed the characteristic bands of the hydroxyl groups at $3387.9 \mathrm{~cm}^{-1}$, ester group at 1734.3 $\mathrm{cm}^{-1}$ and the glycosidic linkage at $1072.7 \mathrm{~cm}^{-}$ 1.34,35 The interglycosic linkages of the saccharide units was confirmed by the downfield shift of C-3 of the inner rhamnose unit in ${ }^{13} \mathrm{C}$-NMR (Table 5) at $\delta 83.59 .{ }^{32-34}$ Therefore compound VII was identified as 3O- $\beta$-D-glucopyranosyl $\quad(1 \rightarrow 3) \quad \alpha$-Lrhamnopyranosyl-quinovic acid-28- $\beta$-Dglucopyranoside.
Compound VIII, exhibited an ester group absorption at $1732.8 \mathrm{~cm}^{-1}$ and hydroxyl group at $3422.2 \mathrm{~cm}^{-1}$ in its IR spectrum. ${ }^{26,34}$ CI-MS investigation of the molecular ion afforded intense daughter ions at $\mathrm{m} / \mathrm{z} 925.8$ [M-Glc] ${ }^{+}$, 880.4 [M-Glc-COO$\left.^{-}\right]^{+}, \quad 749.6$ [M-Glc-Xyl$\left.\mathrm{COO}^{-}\right]^{+}, 719.8\left[\mathrm{M}-2 \mathrm{Glc}^{\left.-\mathrm{COO}^{-}\right]^{+}, 618.7[\mathrm{M}-\mathrm{Glc}-}\right.$ Xyl-GlcA $]^{+}, 587.5$ [M-2Glc-COO-Xyl] ${ }^{+}, 455.4$ $\left[\mathrm{M}^{+}-2 \mathrm{Glc}-\mathrm{Xyl}-\mathrm{GlcA}\right]^{+}$and 411 [M-2Glc-XylGlcA-COO- $]^{+}$. These data suggested that compound VIII consisted of an aglycone with a molecular weight of 455 bound glycosidically through an acyl unit to a sugar chain consisting of one glucosyl unit, a second sugar chain consisting of one xylose, one glucose and one glucuronic acid bound O-glycosidically. ${ }^{25-27}$ This was supported by alkaline hydrolysis which gave D-glucose only as sugar moiety while on acid hydrolysis of compound VIII yielded D-glucose, D-xylose and D-glucuronic acid as sugar moiety. ${ }^{1} \mathrm{H}$ and ${ }^{13} \mathrm{C}-\mathrm{NMR}$ (Table $5)$ spectra showed four signals for anomeric protons $(\delta 4.69,4.80,5.15$ and 5.20) and four anomeric carbons $(\delta 103.34,104.29,104.72$ and 94.80). ${ }^{32,35}$ This confirmed that compound VIII consisted of four sugar moieties. The ${ }^{13} \mathrm{C}$ NMR spectrum of compound VIII showed 53 carbon signals, from which 30 were assigned, to the aglycone part, 23 to the saccharide moiety. The interglycosidic linkages were determined by observing a downfield shift of $\mathrm{C}-2$ and $\mathrm{C}-3$ of inner glucuronic acid in ${ }^{13} \mathrm{C}$ NMR (Table 5) at $\delta 78.60$ and 82.91, this proved that the terminal D-glucose and Dxylose were linked at these carbons. From these results compound VIII was established as 3-O- $\beta$-D-xylopyranosyl- $(1 \rightarrow 3)-[\beta-D-$

glucopyranosyl- $(1 \rightarrow 2)$ ]-oleanolic acid-28-O- $\beta$ D- glucopyranoside.

\section{Bioassay screening}

Screening of the isolated compounds IVIII as molluscicidal agent against $B$. alexandrina snails, the intermediate host of Schistosoma mansoni in Egypt exhibited that only monodesmosidic saponins III and VI have high activities $\left(\mathrm{LC}_{90}=7\right.$ and $\left.6 \mathrm{ppm}\right)$ for the two compounds respectively within 24 hours exposure whereas all other compounds (bidesmosidic saponins and flavonoids glycosides) did not show any activity up to 50 ppm. From these results it appeared that the leaves and stems of $O$. reticulatum have a lot of 
inactive bidesmosidic triterpenoidal saponins such as saponins II, V, VII and VIII. This bidesmosidic saponin content probably hydrolyze to active monodesmosidic saponins when the water suspension of the dry plant powder was molluscicidally tested. ${ }^{8}$ This result is in full agreement with the previous Hosttetmann et al., studies. ${ }^{3,4}$ They reported that bidesmosidic triterpenoidal saponins (have two sugar chains at C-3 and C-28 of the aglycone part) are molluscicidally inactive against different snail species while the monodesmosidic triterpenoidal saponins (have one sugar chain at $\mathrm{C}-3$ of the aglycone part) showed strong potencies against snails. This finding also supports the previous conclusion that explained the absence of any molluscicidal effect of all extracts of this plant. ${ }^{8}$ In this study, it is also evident that the two flavonoid compounds I and IV gave negative results in the bioassay test against the snails. This is in good accordance with previous reports on most flavonoid compounds as they are molluscicidally inactive. ${ }^{7}$

\section{REFERENCES}

1- E. K. Markel, M. Voge and D. T. John, Control of Schistosomiasis in: Medical parasitology $6^{\text {ed. }}$ Saunder W. B. Co. (ed) p. 169 (1986).

2- A. Marston, M. Maillard and K. Hostettmann, J. Ethnopharmacol., 38, 215223 (1993).

3- K. Hostettmann and J. L. Wolfender, Pestic. Sci., 51, 471-482 (1997).

4- S. Y. Liu, F. Sporer, M. Wink, J. Jourdane, R. Henning, Y. L. Li and A. Ruppel, Tropical Medicine and International Health 2, 179-188 (1997).

5- K. Hostettmann, H. Kizu and T. Tomimori, Planta Medica 44, 34-35 (1982).

6- M. M. Abdel-Gawad, M.M. El-Sayed and E. S. Abdel-Hameed, Fitoterapia 70, 371381 (1999).

7- H. Kloos and F. S. McCullough, Plant molluscicide (Mott, K. E., ed.), pp. 45108, John Wiley, Chichester (1987).

8- H. H. El-Nahas, M. M. Abdel-Gawad, M. M. El-Sayed and E. S. Abdel-Hameed, Egypt. J. Biomed. Sci., 9, 28-40 (2002).
9- F. R. Malek, T. Miyase, S. M. AbdelKhalik, M. H. Hetta and I. I Mahmoud, Phytochemistry 60, 185-195 (2002).

10- H. A. Shoeb, I. F. Zeid, L. A. Refahy and A. M. Kalifa, Az. J. Pharm. Sci., 13, 33-40 (1994).

11- R. A. Johnsonand and J. B. Devenpart. Biochemistry and Methodology of Lipids. A division of John Wiley and Sons-Inc New York (1971).

12- A. S. El-Kashoury, Zag. J. Pharm., 3, 116119 (1994).

13- T. I. Khalifa, H. A. Ammer, H. A. Azizi and M. Hosny, Az. J. Nat. Prod., 3, 23-49 (1989).

14- WHO. Molluscicide screening and evaluation. Bull. Wld. Hlth. Org. 33, 567581 (1965).

15- J. T. Litchfield and F. E. Wilcoxon, J. Pharm. Exp. Ther., 96, 99-113 (1949).

16- S. M. Abdel-Wahab, M. A. Selim and N. M. El-Fiki, Bull. Fac. Pharm. Cairo Univ., 28, 67-70 (1990).

17- A. H. Abou-Zeid, N. E. Awad and M. M. El-Missiry, Egypt J. Pharm. Sci., 35, 445452 (1994).

18- T. T. Mabry, K. R. Markham and M. B. Thomas, The Systematic Identification of Flavonoids. Springer Verlag, New York (1970).

19- O. Barbera, J. F. Sanz, N. Sanchez, J. Parareda and J. A. Marco, Phytochemistry 33, 2360-2365 (1986).

20- F. De Simon, A. Dini, C. Pizza, P. Satwnimo and O. Schettino, Phytochemistry 29, 3690-3692 (1990).

21- K. R. Markham, B. Ternai, R. Stanely, H. Geiger and T. J. Mabry, Tetrahedron 34, 1389-1397 (1978).

22- A. Sakushima and S. Nishibe, Phytochemistry 27, 915-919 (1988).

23- L. Rastrelli, P. Santurnino, O. Schettino and A. Dini., J. Agric. Food Chem., 43, 2020-2024 (1995).

24- B. Acebes, M. Bernabe, A. D. Diaz-Lanza and C. Bartolome, J. Nat. Prod., 61, 15571559 (1998).

25- Z. Jia, K. Koike and T. Nikaido, J. Nat. Prod., 61, 1368-1373 (1998).

26- V. D. Huan, S. Yamamura, K. Ohtani, R. Kasai, K. Yamasaki, N. T. Nham, and H. M. Chau, Phytochemistry 47, 451-457 (1998). 
27- G. Carvalho, M. Carvalho and R. BrazFilho, Phytochemistry 52, 1617-1619 (1999).

28- A. Marouf, S. Desbene, T. C. Khanh, H. Wagner, M. Correia, B. Chauffert and M. A. Lacaille-Dubois, Pharmaceutical Biology 39, 263-267 (2001).

29- S. M. Abdel-Khalik, T. Miyase, H. A. ElAshaal and F. R. Melek, Phytochemistry 54, 853-859 (2000).

30- E. Stahl, Chem. Ztg., 82, 323-327 (1958).

31- J. Tian, F. Wu, M. Qiu and R. L. Nie, Phytochemistry 32, 1539-1542 (1993).
32- K. Xiao, Y. H. Yi, Z. Wang, H. Tang, Y. Li and H. W. Lin, J. Nat. Prod., 62, 10301032 (1999).

33- S. Yahara, M. Kohjyouma and H. Kohoda, Phytochemistry 53, 469-471 (2000).

34- R. Aquino, N. De Tommasi, F. De Simone and C. Pizza, Phytochemistry 54, 10351040 (1997).

35- G. Fan and Z. He, Phytochemistry 44, 1139-1143 (1997).

36- H. A. Hassanean, M. A. El-Hammouly, S. A. El-Moghazy and D. W. Bishay, Phytochemistry 33, 667-670 (1993). 